\title{
Phase-I Trigger Readout Electronics Upgrade for the ATLAS Liquid-Argon Calorimeters
}

\author{
Alessandra Camplani ${ }^{12}$ \\ on behalf of the ATLAS Liquid Argon Calorimeter group \\ 1 Dipartimento di Fisica - Universitá degli Studi di Milano \\ 2 I.N.F.N., Milano \\ alessandra.camplani@mi.infn.it
}

\begin{abstract}
The upgrade of the Large Hadron Collider scheduled for the Long Shut-down period of 2019-2020, referred to as Phase-I upgrade, will increase the instantaneous luminosity to about three times the design value. Since the current ATLAS trigger system does not allow sufficient increase of the trigger rate, an improvement of the trigger system is required. The Liquid Argon (LAr) Calorimeter read-out will therefore be modified to use digital trigger signals with a higher spatial granularity in order to improve the identification efficiencies of electrons, photons, tau, jets and missing energy, at high background rejection rates at the Level-1 trigger.
\end{abstract}

\section{Introduction}

The Large Hadron Collider (LHC) has shown very good performances and breaks own records during Run 1 (2009-2013) and Run 2 (2015-2018). In particular, in June 2016, LHC exceeded the peak record instantaneous luminosity of $10^{34}$ $\mathrm{cm}^{-2} \mathrm{~s}^{-1}$. The luminosity value will again increase in the next years. During Run 3 (2021-2023) LHC design parameters should allow for an ultimate peak instantaneous luminosity of $3 \cdot 10^{34} \mathrm{~cm}^{-2} \mathrm{~s}^{-1}$. While during Run 4 (after 2025) an instantaneous luminosity of $5 \cdot 10^{34} \mathrm{~cm}^{-2} \mathrm{~s}^{-1}$ will be delivered. Since ATLAS trigger system does not allow a sufficient increase of the trigger rate, an electronic upgrade is required [1].

\section{ATLAS Liquid Argon (LAr) calorimeter Upgrade}

To face the Run 3 luminosity, the LAr calorimeter trigger electronics will be modified, in order to maintain a low $p_{T}$ lepton threshold and keep the same trigger bandwidth with respect to Run 2 . The new trigger readout electronics will be installed during the second Long Shut-down (LS2). The aim is to provide higher-granularity, higher-resolution and longitudinal shower information from the calorimeter to the Level-1 trigger processors. Figure 1 compares the electron energy deposition in the present system to the new proposed system, which has ten time finer granularity in the trigger readout from the calorimeter. The 


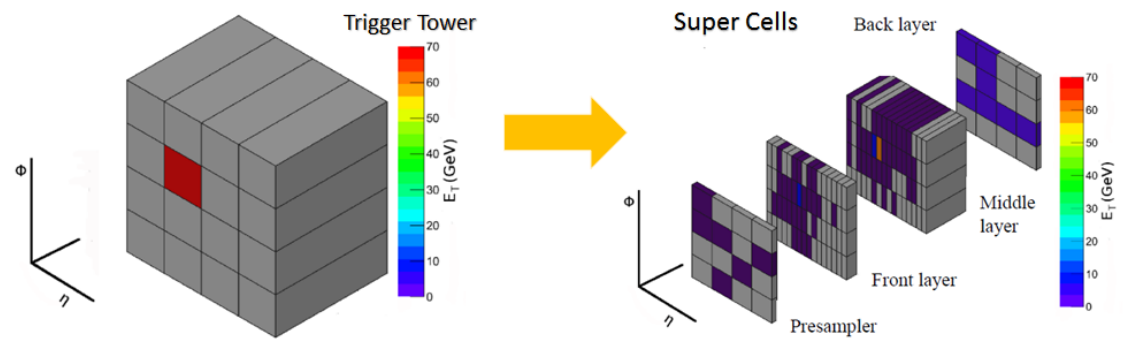

Fig. 1: Trigger signal granularity improvement from Trigger Towers $(\Delta \eta \times \Delta \phi=0.1 \times$ $0.1)$ to Super Cells $(\Delta \eta \times \Delta \phi=0.025 \times 0.1$ in front and middle layers $)$.

existing calorimeter trigger readout, the so-called Trigger Tower, will evolve in the new finer granularity scheme, called Super Cells (SC). In total there will be 34000 SCs [2].

\subsection{LAr Front-End (FE) Electronics}

The LAr FE electronics will be improved and extended during the Phase-I Upgrade. A new Layer Sum Board (LSB) will produce the finer granularity SC signals in the Front and Middle layers. A new baseplane will keep the compatibility with the existing setup and will route the new signals. And a new LAr Trigger Digitizer Boards (LTDB) will receive, digitize and send to the BE electronics the SC signals. The LTDB, shown in Fig. 2, is the key board for the Phase-I Upgrade. There will be 124 LTDB boards and each of them will process up to $320 \mathrm{SC}$ analog signals. As the LTDB will be exposed to a high radiation level, all components, especially newly developed ASICs, have to be subjected to extensive radiation qualification tests [3].

The custom ADC is a quadchannel 12-bit $40 \mathrm{MS} / \mathrm{s}$ pipeline SAR ADC, designed with $130 \mathrm{~nm}$ technology [4]. Recently, the radiation tolerance of the prototype ADC chip has been tested and confirmed up to 10 MRad (the requirement is $100 \mathrm{kRad}$ ).

LOCx 2 is a dual $8 \times 12$ bit serializer, with 5.12 Gbps of output [5]. LOCld is a VCSEL (Vertical Cavity Surface-Emitting Lasers) driver, designed for the optical interface [6]. Both are fabricated with $250 \mathrm{~nm}$

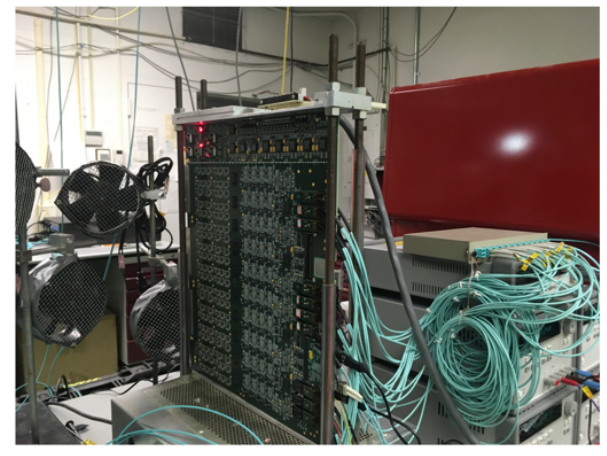

Fig. 2: LTDB board under test.

Silicon-on-Sapphire CMOS technology and have been irradiated up to $200 \mathrm{kRad}$. No changes in the output eye diagrams have been observed. 


\subsection{LAr Back-End (BE) Electronics}

The LAr calorimeter BE electronics system, called the LAr Digital Processing System (LDPS), will receive digital SC data from the LTDBs, reconstruct $E_{T}$ (the transverse energy of each SC) and transmit the results to the Level-1 calorimeter trigger system every 25 ns. The LDPS consists of 32 ATCA Car-

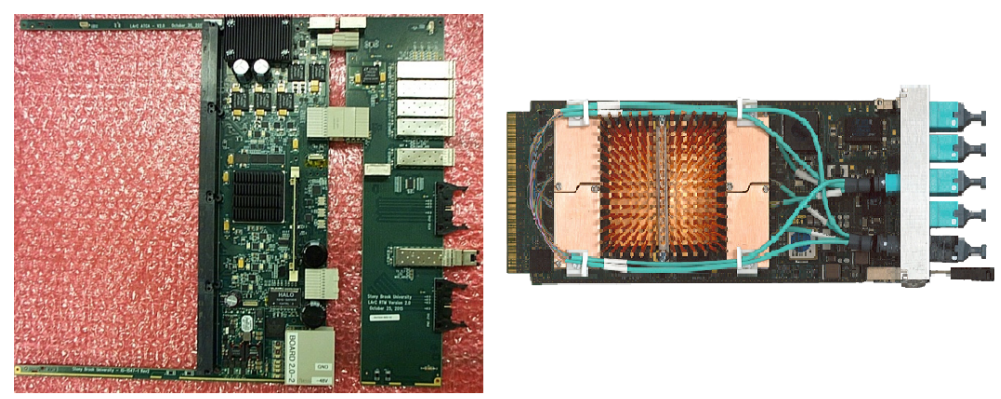

Fig. 3: ATCA Carrier and LATOME board.

rier boards, each one equipped with four Advanced Mezzanine Cards (AMC) called LATOME, both shown in Fig. 3. The LATOME is built around a highperformance and very competitive FPGA, an ARRIA-10 from Intel FPGA. The main data path is passing through the LATOME where the transverse energy of each SC is built.

A System Test with the final prototype is being prepared. The first tests were done at the beginning of 2017 , and more are planned for the next months. The purpose is to confirm all the functionalities and stabilities before the mass production.

\section{Demonstrator}

A demonstrator of the Phase-I upgrade was installed in ATLAS, during summer 2014, in the calorimeter ElectroMagnetic (EM) barrel, with a coverage of $1 / 32$ of barrel region, to show the feasibility of the Phase-I Upgrade.

The demonstrator is reading data from the Super Cells with the aim of validating the energy reconstruction and collecting real collision data for the filtering algorithm development. Of course this will allow to gain some experience in the installation and operation of such equipment in the ATLAS environment.

In the FE, two LTDBs are installed to digitize the calorimeter data, from 284 Super Cells in the EM barrel, and transmit them along 48 optical links at 4.8 Gbps to three BE boards called ABBA (ATCA test Board for Baseline Acquisition), with a total throughput of about 200 Gbps per LTDB. Each ATCA board mounts three Stratix-IV Intel FPGAs. Two FPGAs store the SC data inside circular buffers and wait for a Level-1 trigger to select the interesting event. The 
third FPGA takes care of the readout via IPbus protocol over UDP on $10 \mathrm{GbE}$ network.

The demonstrator system had a successful data taking during 2015 and 2016, collecting data in parallel with ATLAS for both protonproton and heavy ion collisions. This can be done also thanks to the special Level-1 topological trigger item provided by the Level-1 community that is allowing to compared the demonstrator readout with the ATLAS main readout. Next step is to have a final PhaseI prototype (LTDB and LATOME) installed and running at the end of this year and oper-

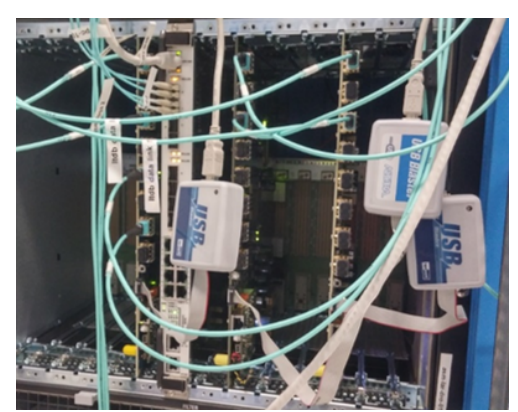

Fig. 4: ABBA boards. ate until the end of Run- 2 .

\section{Conclusion}

The ATLAS LAr calorimeter electronics will be upgraded for Phase-1 after LS2 (2019-2020). The calorimeter trigger path will be digitized at FE level, to make use of an improved granularity for the trigger decision. Presently, the new FE and BE systems are being developed and produced. The LTDB prototype is being assembled, the radiation tolerant ASICs have been designed and they are under test as well as the LDPB ATCA carrier and LATOME AMC boards. In the meantime, a demonstrator has been installed on the detector, during summer 2014. During 2015 and 2016 it has collected data in parallel with ATLAS for both proton-proton and heavy ion collisions and now it's getting ready also for 2017 data. This demonstrator is giving the possibility to gain some experience in order to be prepared for the final prototype installation and runs in 2018.

\section{References}

1. ATLAS Collaboration, "The ATLAS Experiment at the CERN Large Hadron Collider", JINST 3 (2008) S08003.

2. M. C. Aleksa et al., "ATLAS Liquid Argon Calorimeter Phase-I Upgrade Technical Design Report," CERN-LHCC, https://cds.cern.ch/record/1602230.

3. N. J. Buchanan et al., "Radiation qualification of the front-end electronics for the readout of the ATLAS liquid argon calorimeters", JINST 3 (2008) P10005.

4. H. $\mathrm{Xu}$, "The Trigger Readout Electronics for the Phase-I Upgrade of the ATLAS Liquid Argon Calorimeters", ATL-LARG-PROC-2016-003, https://cds.cern.ch/record/2229579.

5. L. Xiao et al., "LOCx2, a low-latency, low-overhead, 2 5.12-Gbps transmitter ASIC for the ATLAS Liquid Argon Calorimeter trigger upgrade", JINST 11 (2016) C02013.

6. F Liang and others, "The design of 8-Gbps VCSEL drivers for ATLAS liquid Argon calorimeter upgrade", JINST 8 (2013) C01031.

(C) 2017 CERN for the benefit of the ATLAS Collaboration.

Reproduction of this article or parts of it is allowed as specified in the CC-BY-4.0 license. 